\title{
COVID-19 quarantine: Two-way interaction between physical activity and mental health
}

\author{
Mohsen Khosravi \\ Department of Psychiatry and Clinical Psychology, Zahedan University of Medical Sciences, \\ Zahedan, Iran \\ This article is distributed under the terms of the Creative Commons Attribution Noncommercial License (CC BY-NC 4.0) which permits \\ any noncommercial use, distribution, and reproduction in any medium, provided the original author(s) and source are credited.
}

\begin{abstract}
Recent studies have revealed that physical activity significantly reduces the risk of coronavirus disease 2019 (COVID-19) infection by strengthening the immune system. Also, regular physical activity can reduce the risks of developing physical and mental health problems such as diabetes, hypertension, coronary heart disease, stress, anxiety, depression, etc. However, the two-way interaction between physical activity and psychological symptoms has not been well addressed yet. This paper is intended to examine various dimensions of this interaction and its effects on mental health at the time of COVID-19 quarantine.
\end{abstract}

Key Words: COVID-19, mental health, physical activity.

Eur J Transl Myol 2020; 30 (4): 9509. doi: 10.4081/ejtm.2020.9509

Coronavirus disease 2019 (COVID-19) outbreak was first reported in late December 2019 in Wuhan, China. ${ }^{1,2}$ It was recognized by the World Health Organization (WHO) in January 2020 as a Public Health Emergency of International Concern. The rapid spread of the COVID-19 to other countries worldwide prompted the WHO to announce this crisis as a global pandemic in March 2020. ${ }^{3}$ Soon or later, countries implemented early containment strategies, such as: i) home isolation for those in 'high-risk' groups (e.g., $\geq 70$ years old); ii) 14day self-isolation for people who were suspected or confirmed to have COVID-19 infection; iii) keeping 2 meters physical distance from one another; iv) closures of schools and childcare providers; v) closures of services and amenities (bars, pubs, restaurants, gyms, pools, etc.) and vi) implementing dedicated hospital services, i.e., COVID-19 diagnostics, ${ }^{4-9}$ clinics and intensive car units to stop the virus from spreading through the population and minimizing the burden of COVID-19 crisis on population and on national healthcare systems. ${ }^{10}$ Although these measures have been associated with a reduced transmission chain of COVID-19, they have highly overshadowed the physical and mental health of individuals. ${ }^{11,12}$ Nevertheless, the impacts of obliged pandemic-related behavioral changes on these aspects of people lives have not been well discussed. Only a few recent reports demonstrated that strategies to reduce spreading COVID-19 affect levels of physical activity ${ }^{13-}$ 15 and psychological symptoms. ${ }^{11,15,16}$ In detail, during quarantine time, physical activity levels appear to decrease while the amount of time spent in sedentary behaviors potentially increases due to limited opportunities for activity. ${ }^{17}$ Besides, the recent COVID19 disaster has posed major challenges for maintaining people's mental health. ${ }^{18}$ As a result, both people with or without a history of mental illness may experience high levels of distress and double pressure at the time of the COVID-19 pandemic, particularly in isolation. ${ }^{19}$ For instance, a 2020 rapid review indicated that quarantine causes acute negative psychological effects with potentially long-term consequences. ${ }^{20}$ Several recent studies on adults in all parts of the world have revealed high levels of depressive and anxiety symptoms, probably associated with the COVID-19 pandemic. ${ }^{11}$

Since current empirical evidence suggested positive benefits of regular physical activity and physical exercise to mental health outcomes of COVID-19 quarantine, a sedentary lifestyle may serve to complicate an alreadyproblematic situation. ${ }^{17,21-24}$ In other words, exercise is a control mechanism for many mentally ill people and a protective factor for those without any psychological disorders. ${ }^{19}$ Some of the positive effects of regular physical activity on psychological health include: (i improving one's self-esteem and a sense of well-being; (ii reduction of stress, anxiety, and depression by making changes in the hypothalamic-pituitary-adrenal axis and mediation of the endogenous opioid system; and (iii upregulation of different trophic factors, such as brainderived neurotrophic factor. ${ }^{22}$ A recent study has recommended that the time required for exercising 
Table 1. Summaries of psychological techniques for increasing physical activity during quarantine time (adapted from Diamond \& Waite, 2020) ${ }^{23}$
Beliefs about activity
Identification of cognitive biases
Cognitive biases are systematic errors in thinking that may occur during the processing and interpretation of surrounding events and affect people's decisions and judgments.
Cognitive restructuring
Cognitive reconstruction is a psychotherapeutic technique of learning to identify and cope with maladaptive and irrational thoughts, called cognitive distortions.
Socratic dialogue
This technique, known by various names such as Socratic questioning, Socratic method, and Maieutic method, is one of the methods used by psychologists in exploring the mind to help people discover new aspects of thinking about self and the world.
Behavioral experiments
Behavioral experiments are planned experiential activities to test the validity of individuals' beliefs about self, others, and the world, or to test new and more adaptive beliefs.
Savoring
Savoring comprises the special focusing on pleasurable aspects before, during, or after an activity. Psychotherapists can use these techniques to evoke this memory in mind and generate motivation before repeating the activity.
Imagery
Creating an image of task completion can increase the involvement likelihood by reducing the power of unhelpful images and building self-confidence.

\section{Motivation for activity}
Goal-setting
Mental contrasting
Motivational interviewing
Goal-setting is the act of selecting a specific, realistic, and time-based target that one wishes to achieve. Recent studies have proposed that performance goals (e.g., achieving 10,000 steps per day) are probably less helpful to those starting an activity compared to the goals directed toward learning new strategies (e.g., finding four ways to increase step count during the week).
Mental contrasting is a self-regulatory strategy that contributes to the commitment to the goal. For instance, "Although I am not allowed to leave the house, I still exercise by watching online sports videos".
Imagining a different future
Motivational interviewing is a client-centered counseling style to extract behavior change by helping the client discover and resolve ambivalence. This is especially important given the additional barriers imposed by the pandemic, including limited access to facilities and group activities.
Imagining a different future In this method, clients are asked to imagine that they are able to live without current obstacles following a miracle.
Psychoeducation
Psychoeducation about the beneficial effects of exercise on psychological symptoms can help increase physical activity during the quarantine period.
The sense of reward obtained
from the activity
Behavioral interventions
Psychologists can use step-by-step planning to help people choose the right amount of activity for themselves and explore ways to ensure that the experience is rewarding and not aversive.
Problem-solving
This method involves producing several possible solutions alongside their advantages and disadvantages, which can facilitate the selection and testing of a solution and eliminate the feeling of being trapped in the deadlock. Identifying innovative ways to work out at home, such as accessing online sports classes, or learning how to keep distancing while exercising outdoors, are examples that can be resolved using this method.

during the quarantine would be at least 30 minutes of moderate-intensity or 20 minutes of vigorous-intensity aerobic exercise training every day. ${ }^{25}$

Although the effects of exercise on mental health can be enhanced by increasing its intensity, the intensity and volume of exercise should be adjusted and controlled separately for each person using professional counseling, for the sake of stimulus effectiveness. ${ }^{26}$ Reciprocally, psychological barriers have long been realized as significantly important and decisive factors to prevent activity, especially among people with mental health problems. Additionally, during a pandemic, there may be additional barriers to increased activity, such as anxiety about leaving home, the closure of gym facilities, and less chance of group exercise..$^{23}$ Psychologically, three cognitive factors may be involved in an activity: (i) beliefs about the activity; (ii) motivation for the activity; and (iii) the sense of reward obtained from the activity. According to psychological theory, this complex set of considerations can be used in a meaningful psychological approach through an individualized formulation. ${ }^{23}$

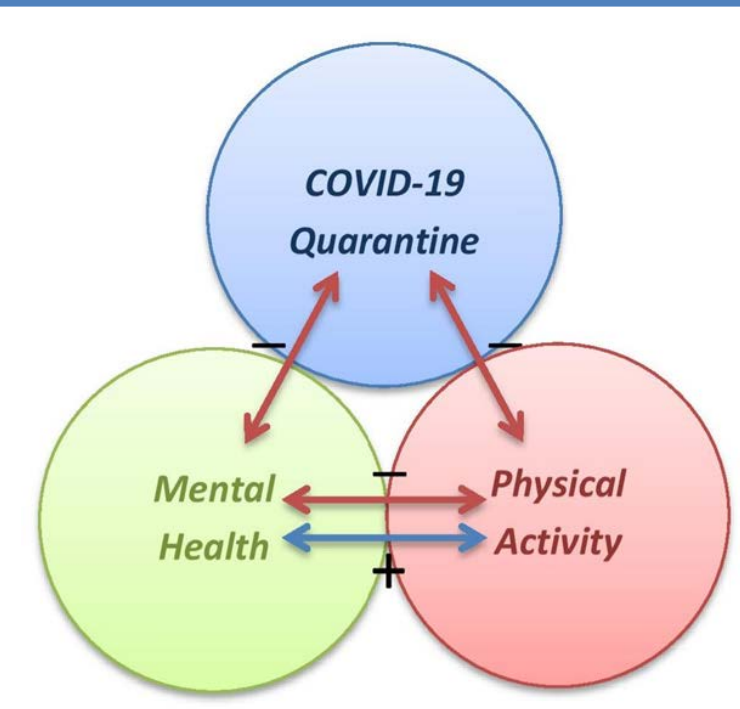

Fig 1. Interactions between COVID-19 quarantine, physical activity, and mental health 
For example, as regards beliefs about physical activity, Beck's cognitive model describes how people's beliefs about the self, others, and the world may inhibit physical activity and are thus a key target in activity-enhancing interventions. ${ }^{27}$ Also, as to motivation, psychoeducation can increase people's awareness of the beneficial effects of physical activity on mood and anxiety, and encourage them to make lifestyle changes. ${ }^{28}$ Table 1 summarizes other psychological techniques in this area. ${ }^{23,29,30}$

In conclusion, given the two-way interaction between physical activity and psychological symptoms (Figure 1), clinicians should assume them as a treatable clinical goal, depending on the circumstances. Moreover, due to the different benefits that physical activity provides to people's mental health, in any legal statement issued by government agencies, physical training, both indoors and outdoors, should be considered an essential application during the COVID-19 periods of isolation and quarantine.

\section{List of acronyms}

COVID-19 - Coronavirus disease 2019

WHO - World Health Organization

\section{Authors contributions}

Mohsen Khosravi fully wrote the article.

\section{Acknowledgments}

None.

\section{Funding}

None.

\section{Conflict of Interest}

The author declare no financial, personal, or other conflicts of interest.

\section{Ethical Publication Statement}

The author confirms that he has read the journal's position on the issues involved in ethical publishing and states that this report is consistent with those guidelines.

\section{Corresponding Author}

Mohsen Khosravi, MD, Department of Psychiatry and Clinical Psychology, Baharan Psychiatric Hospital, Zahedan University of Medical Sciences, Postal Code: 9813913777, Zahedan, Iran.

Tel: +98-5433522636, Fax: +98-54335183527.

ORCID iD: 0000-0003-2970-6309

Email: dr_khosravi2016@yahoo.com

\section{References}

1. Carraro U. 2020PMD, 30-years of Translational Mobility Medicine at the time of COVID-19 outbreak: Last-minute forewords from the editor. Eur J Transl Myol 2020;30(1):8966. doi: 10.4081/ ejtm.2019.8966

2. Angelini C, Siciliano G. Neuromuscular diseases and COVID-19: Advices from scientific societies and early observations in Italy. Eur J Transl Myol 2020;30(2):9032. doi: 10.4081/ejtm.2019.9032

3. Khosravi M. Stress reduction model of COVID-19 pandemic. Iran J Psychiatry Behav Sci 2020;14(2):e103865. doi: 10.5812/ijpbs.103865

4. Vashist SK. In Vitro Diagnostic Assays for COVID-19: Recent Advances and Emerging Trends. Diagnostics 2020;10(4):202. doi: 10.3390/ diagnostics10040202

5. Kontou PI, Braliou GG, Dimou NL, et al. Antibody Tests in Detecting SARS-CoV-2 Infection: A MetaAnalysis. Diagnostics 2020;10(5):319. doi: 10.3390/diagnostics10050319

6. Mashamba-Thompson TP, Crayton ED. Blockchain and Artificial Intelligence Technology for Novel Coronavirus Disease 2019 Self-Testing. Diagnostics 2020;10(4):198. doi: 10.3390/ diagnostics10040198

7. Kubina R, Dziedzic A. Molecular and Serological Tests for COVID-19. A Comparative Review of SARS-CoV-2 Coronavirus Laboratory and Pointof-Care Diagnostics. Diagnostics 2020;10(6):434. doi: 10.3390/diagnostics10060434

8. Allam M, Cai S, Ganesh S, et al. COVID-19 Diagnostics, Tools, and Prevention. Diagnostics 2020;10(6):409. doi.org/10.3390/diagnostics10060409

9. Han P, Ivanovski S. Saliva - Friend and Foe in the COVID-19 Outbreak. Diagnostics 2020;10:290. doi:10.3390/diagnostics10050290

10. Nussbaumer-Streit B, Mayr V, Dobrescu AI, et al. Quarantine alone or in combination with other public health measures to control COVID-19: a rapid review. Cochrane Database Syst Rev 2020;4:CD013574. doi: 10.1002/14651858.CD013574

11. Kontoangelos K, Economou M, Papageorgiou C. Mental health effects of COVID-19 pandemia: a review of clinical and psychological traits. Psychiatry Investig 2020;17:491-505. doi: 10.30 73/pi.2020.0161

12. Moro T, Paoli A. When COVID-19 affects muscle: effects of quarantine in older adults. Eur J Transl Myol 2020;30(2):9069. doi: 10.4081/ejtm. 2019.9069

13. Peçanha T, Goessler KF, Roschel H, et al. Social isolation during the COVID-19 pandemic can increase physical inactivity and the global burden of cardiovascular disease. Am J Physiol Heart Circ Physiol 2020;318:H1441-H1446. doi: 10.1152/ ajpheart.00268.2020

14. Lesser IA, Nienhuis CP. The Impact of COVID-19 on Physical Activity Behavior and Well-Being of Canadians. Int J Environ Res Public Health 2020;17:3899. doi: 10.3390/ijerph17113899

15. Duncan GE, Avery AR, Seto E, et al. Perceived change in physical activity levels and mental health during COVID-19: Findings among adult twin pairs. PLoS One 2020;15:e0237695. doi: 10.1371/ journal.pone.0237695 


\section{COVID-19 quarantine: physical activity and mental health}

Eur J Transl Myol 2020; 30 (4): 9509. doi: 10.4081/ejtm.2020.9509

16. Tian F, Li H, Tian S, et al. Psychological symptoms of ordinary Chinese citizens based on SCL-90 during the level I emergency response to COVID19. Psychiatry Res 2020;288:112992. doi: 10.1016/ j.psychres.2020.112992

17. Schuch FB, Bulzing RA, Meyer J, et al. Associations of moderate to vigorous physical activity and sedentary behavior with depressive and anxiety symptoms in self-isolating people during the COVID-19 pandemic: A cross-sectional survey in Brazil. Psychiatry Res 2020;292:113339. doi: 10.1016/j.psychres.2020.113339

18. Zarghami M. Psychiatric Aspects of Coronavirus (2019-nCoV) Infection. Iran J Psychiatry Behav Sci 2020;14(1):e102957. doi: 10.5812/ijpbs.102957

19. Morrey LB, Roberts WO, Wichser L. Exerciserelated Mental Health Problems and Solutions during the COVID-19 Pandemic. Curr Sports Med Rep 2020;19:194-5. doi: 10.1249/JSR.00000000 00000725

20. Brooks SK, Webster RK, Smith LE, et al. The psychological impact of quarantine and how to reduce it: rapid review of the evidence. Lancet 2020;395:912-20. doi: 10.1016/S0140-6736(20)30460-8

21. Jiménez-Pavón D, Carbonell-Baeza A, Lavie CJ. Physical exercise as therapy to fight against the mental and physical consequences of COVID-19 quarantine: Special focus in older people. Prog Cardiovasc Dis 2020;63:386-8. doi: 10.1016/j.pcad 2020.03.009

22. Maugeri G, Castrogiovanni P, Battaglia G, et al. The impact of physical activity on psychological health during COVID-19 pandemic in Italy. Heliyon 2020;6:e04315. doi: 10.1016/j.heliyon.2020.e04315

23. Diamond R, Waite F. Physical activity in a pandemic: A new treatment target for psychological therapy. Psychol Psychother 2020;10.1111/papt.12294. doi: 10.1111/papt.12294

24. Jacob L, Tully MA, Barnett Y, et al. The relationship between physical activity and mental health in a sample of the UK public: A crosssectional study during the implementation of COVID-19 social distancing measures. Ment Health Phys Act 2020;19:100345. doi: 10.1016/ j.mhpa.2020.100345

25. Chen P, Mao L, Nassis GP, Harmer P, Ainsworth BE, Li F. Coronavirus disease (COVID-19): The need to maintain regular physical activity while taking precautions. J Sport Health Sci 2020;9:1034. doi: 10.1016/j.jshs.2020.02.001

26. Amatriain-Fernández S, Murillo-Rodríguez ES, Gronwald T, Machado S, Budde H. Benefits of physical activity and physical exercise in the time of pandemic. Psychol Trauma 2020;12:S264-S266. doi: 10.1037/tra0000643

27. Beck AT. Depression: Clinical, experimental, and theoretical aspects. New York: Hoeber Medical Division, Harper \& Row; 1967.

28. Hearon BA, Beard C, Kopeski LM, Smits JA, Otto MW, Björgvinsson T. Attending to timely contingencies: promoting physical activity uptake among adults with serious mental illness with an exercise-for-mood vs. an exercise-for-fitness prescription. Behav Med 2018;44:108-15. doi: 10.1080/08964289.2016.1276428

29. Swann C, Rosenbaum S, Lawrence A, Vella SA, McEwan D, Ekkekakis P. Updating goal-setting theory in physical activity promotion: a critical conceptual review. Health Psychol Rev 2020;1-17. doi: 10.1080/17437199.2019.1706616

30. Kwasnicka D, Ntoumanis N, Sniehotta FF. Setting performance and learning goals is useful for active and inactive individuals, if goals are personalized and flexible: commentary on Swann et al. (2020). Health Psychol Rev 2020:1-5. doi: 10.1080/ 17437199.2020.1762107

Submission: September 13, 2020 Accepted for pubblication: September 28, 2020 